\title{
Bounds on Volume Increase Under Dehn Drilling Operations
}

\author{
Martin Bridgeman * \\ September 6, 2018
}

\begin{abstract}
In this paper we investigate how the volume of hyperbolic manifolds increases under the process of removing a curve, that is, Dehn drilling. If the curve we remove is a geodesic we are able to show that for a certain family of manifolds the volume increase is bounded above by $\pi \cdot l$ where $l$ is the length of the geodesic drilled. Also we construct examples to show that there is no lower bound to the volume increase in terms of a linear function of a positive power of length and in particular volume increase is not bounded linearly in length.
\end{abstract}

\section{Introduction}

Let $M$ and $M^{\prime}$ be three-dimensional manifolds such that $M^{\prime}$ is homeomorphic to $M-\alpha$, where $\alpha$ is a simple closed curve in $M$. Then we say that $M^{\prime}$ is obtained by Dehn drilling on $M$ and $\alpha$ is the curve drilled.

If $M$ and $M^{\prime}$ are complete hyperbolic manifolds of finite volume then Gromov ([Th]) showed that the volume of $M^{\prime}$ is greater than the volume of $M$. Therefore hyperbolic Dehn drilling always increases volume, but what this increase depends on is unknown. In the following we study the case of drilling out a geodesic and show how the volume increase is related to the length of the geodesic drilled.

Conjecture: 1 Dehn drilling a geodesic increases volume by at most $\pi \cdot L$ where $L$ is the length of the geodesic to be drilled.

In this paper we will prove this conjecture for a class of Dehn drillings as well as give motivation for why the conjecture should be true in general. We also show that in general there is no linear lower bound on the volume increase by showing that there is no linear lower bound for the set of Dehn drillings we consider.

${ }^{*}$ Research at MSRI is supported in part by NSF grant DMS-9022140. 


\section{Motivation}

The motivation for the conjecture and the idea behind the proof in the special case to be considered is the following. If $M^{\prime}$ is obtained by hyperbolic Dehn drilling a geodesic $\alpha$ in $M$ then we first construct a continuous one-parameter family of hyperbolic cone-manifolds $M_{\theta}, 0<\theta \leq 2 \pi$ interpolating between $M$ and $M^{\prime}$. By interpolating we mean that $M_{2 \pi}=M$, $\lim _{\theta \rightarrow 0} M_{\theta}=M^{\prime}$ and $M_{\theta}$ is homeomorphic to $M$ with cone axis $\alpha$ and cone angle $\theta$.

Having constructed a one-parameter family of cone-manifolds interpolating between $M$ and $M^{\prime}$, we apply results of Craig Hodgson ([Ho]) which describe the derivative of volume of a smooth one-parameter family of cone manifolds.

Before we give Hodgson's formula, we state the Sclafli formula for the variation of volume of a one-parameter family of polyhedra in a space of constant curvature, on which Hodgson's formula is based.

Theorem 1 (Sclafli, Hodgson [Ho]) Let $X_{t}$ be a smooth one-parameter family of polyhedra in a simply connected $n$-dimensional space of constant curvature $K$. Then the derivative of the volume of $X_{t}$ satisfies the equation:

$$
(n-1) K d \operatorname{Vol}\left(X_{t}\right)=\sum_{F} V_{n-2}(F) d \phi_{F}
$$

where the sum is over all co-dimension two faces of $X_{t}, V_{n-2}$ is the $(n-2)$-dimensional volume and $\phi_{F}$ is the dihedral angle at $F$.

In the cone manifold case we have the following.

Theorem 2 (Hodgson[Ho]) Let $C_{t}$ be a smooth family of (curvature $K$ ) cone-manifold structures on a manifold with fixed topological type of singular locus. Then the derivative of volume of $C_{t}$ satisfies

$$
(n-1) K d \operatorname{Vol}\left(C_{t}\right)=\sum_{\Sigma} V_{n-2}(\Sigma) d \phi_{\Sigma}
$$

where the sum is over all components $\Sigma$ of the singular locus of $C$ and $\theta_{\Sigma}$ is the cone angle along $\Sigma$.

Therefore in our case where the singular locus is a single cone axis $\alpha$ we have

$$
\begin{aligned}
d V & =-\frac{1}{2} l(\theta) d \theta \\
\Delta V & =\frac{1}{2} \int_{0}^{2 \pi} l(\theta) d \theta
\end{aligned}
$$

where $\theta$ is the cone angle along $\alpha, l(\theta)$ is the length of $\alpha$ in $M_{\theta}$ and where $\Delta V$ is the volume increase under Dehn drilling. 


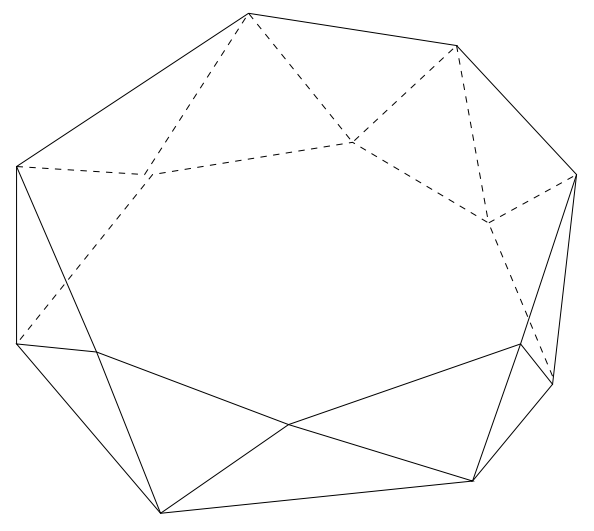

Figure 1: Ideal hyperbolic drum

Therefore if the function $l$ is monotonically increasing in $\theta$ then it follows that

$$
\Delta V \leq \pi \cdot L
$$

where $L$ is the length of the geodesic $\alpha$ in $M$.

The conjecture and the proof of the conjecture for the class of Dehn drillings we consider, is based on this two-fold approach of interpolation and monotonicity.

\section{Basic Polyhedra}

We will now prove that for a Dehn drilling operation on basic polyhedra, the conjecture is true. This Dehn drilling operation has some interesting properties including giving a new way to enumerate all basic polyhedra and hence all link projections ([B2]). A basic polyhedron is an ideal hyperbolic polyhedron $P$ with all dihedral angles being right angles. Therefore all vertices have valence four. Let $\mathcal{B}$ be the set of all basic polyhedra. Examples of basic polyhedra are the ideal hyperbolic drums $\left\{T_{n}\right\}_{n \geq 3}$ which have two faces being regular ideal $n$-gons, corresponding to the top and bottom of the drum and every other face being an ideal triangle (figure 1).

As all the dihedral angles are $\pi / 2$, basic polyhedra can also be thought of as orbifolds. In [B1] it was shown that if $P$ is a basic polyhedron then it has a four-fold cover (in the orbifold sense) $L_{P}$ which is a hyperbolic link complement in $S^{3}$ having a boundary component for each vertex of $P$.

\section{Surgery and Dehn drilling}

If $P$ is a basic polyhedron and $F$ is a non-triangular face of $P$ then we define surgery on $P$ as follows. Choose two non-adjacent edges $e_{1}, e_{2}$ of $F$ and pinch them together to form a new combinatorial polyhedron $\bar{P}$ (figure 2). By Andreev's theorem ([Th]), $\bar{P}$ has a realization $P^{\prime}$ 

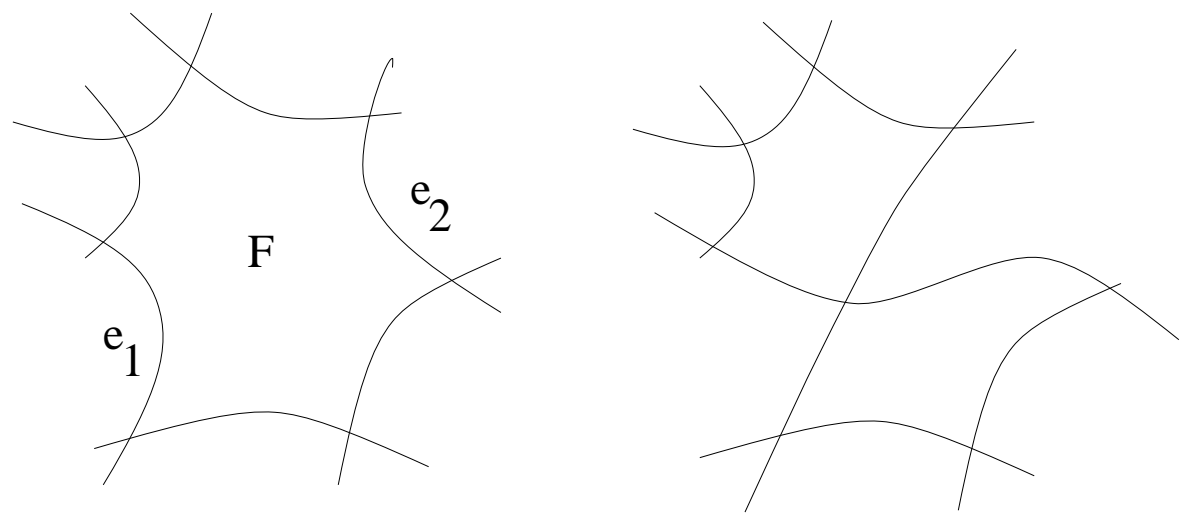

Figure 2: Surgery

which is an ideal hyperbolic polyhedron with all dihedral angles right angles and therefore is also a basic polyhedron. We say $P^{\prime}$ is obtained by surgery on $P$ and therefore surgery is an operation defined on the set $\mathcal{B}$.

In the sense of orbifolds, $P^{\prime}$ is obtained by Dehn drilling the unique geodesic perpendicular to both $e_{1}$ and $e_{2}$ in $P$ and if we lift to the four-fold covers, we see that $L_{P^{\prime}}$ is obtained by Dehn drilling a simple closed geodesic in $L_{P}([\mathrm{~B} 2])$. Thus surgery corresponds to a Dehn drilling operation.

We define a partial ordering on $\mathcal{B}$ by saying $P \prec P^{\prime}$ if $P^{\prime}$ is the result of repeated surgeries starting with $P$. This partial ordering has as initial objects the set $\left\{T_{n}\right\}_{n \geq 3}$ mentioned above ([B2]) and therefore we can enumerate all basic polyhedra by repeated surgery on these initial objects.

Theorem 3 Surgery increases volume by at most $\pi \cdot L$ where $L$ is the length of the geodesic drilled.

To prove this theorem we must first interpolate between $P$ and $P^{\prime}$ where $P^{\prime}$ is obtained by surgery on $P$.

\section{$3 \quad$ Interpolating Surgery}

Let $P$ be a basic polyhedron with $F$ a non-triangular face of $P$ and $e_{1}, e_{2}$ be two nonadjacent edges of $F$. Also let $g$ be the unique geodesic in $F$ perpendicular to $e_{1}, e_{2}$. To interpolate between $P$ and $P^{\prime}$ we need a continuous one-parameter family of polyhedra $P_{\theta}, 0<\theta \leq \pi$ which have combinatorial type of $P \cup\{g\}$, with dihedral angle $\theta$ along $g$ and all other edges having the same dihedral angles as the corresponding edge of $P$ and with $P_{\pi}=P, \lim _{\theta \rightarrow 0} P_{\theta}=P^{\prime}$. Intuitively $P_{\theta}$ is obtained by bending $P$ along $g$ an amount $\pi-\theta$. To find such a one-parameter family of polyhedra we use results of Hodgson and Rivin ([HR]) which characterizes compact hyperbolic polyhedra in terms of their spherical duals. 

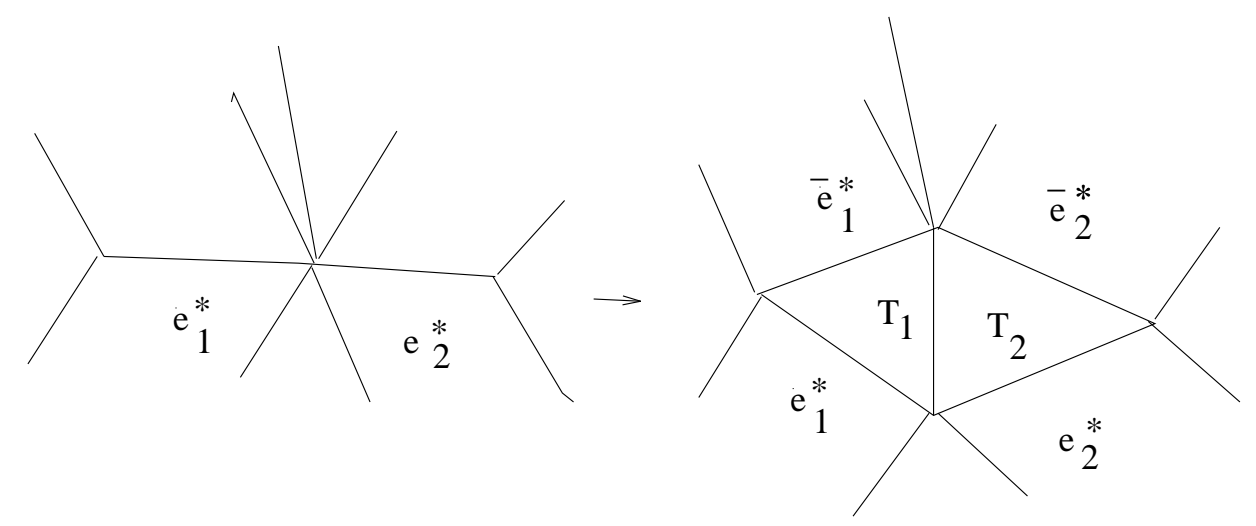

Figure 3: Cutting open $S$ to make $S_{\theta}$

\section{Spherical Dual of a Polyhedron}

If $P$ is a hyperbolic polyhedron then we form its spherical dual $P^{*}$ which is a spherical complex combinatorially dual to the polyhedron $P$ as follows. Each vertex corresponds to a spherical polygon with edges having lengths being the exterior dihedral angles of the edges incident to the vertex and with face angles being the complementary angles of the face angles at the vertex (complement of $\alpha=\pi-\alpha$ ). Similarly an ideal vertex corresponds to a spherical hemisphere with vertices dividing the equator into arcs of length less than $\pi$. Let $S=P^{*}$ and define spherical complexes $S_{\theta}$ by cutting $S$ open at $e_{1}^{*}, e_{2}^{*}$ (dual edges to $e_{1}, e_{2}$ ), and attaching a spherical bigon $B$ with angle $\pi-\theta$. $B$ is further divided into two triangles $T_{1}, T_{2}$ by joining the two points on the equator by an edge $g^{*}$. If $P_{\theta}$ is realisable as a hyperbolic polyhedron then its spherical dual is $S_{\theta}$ with $g^{*}$ dual to $g$. Note that each of $e_{1}^{*}, e_{2}^{*}$ split into two edges $e_{i}^{*}, \bar{e}_{i}^{*}, i=1,2$ (figure 3 ). Thus we have a continuous one-parameter family of spherical complexes $S_{\theta}, 0 \leq \theta \leq \pi$ which interpolates between the spherical duals of $P$ and $P^{\prime}$. Before we can interpolate between $P$ and $P^{\prime}$ we need to know more about the map between hyperbolic polyhedra and spherical complexes.

The following theorem due to Craig Hodgson and Igor Rivin gives a characterization of compact hyperbolic polyhedra in terms of their spherical duals.

Theorem 4 ([HR]) A metric space $(M, g)$ homeomorphic to $S^{2}$ can arise as the spherical dual $P^{*}$ of a compact hyperbolic polyhedron $P$ in $H^{3}$ if an only if the following hold.

(a) The metric has constant curvature 1 away from a finite collection of cone points $c_{i}$.

(b) The cone angles at $c_{i}$ are greater than $2 \pi$.

(c) The lengths of closed geodesics of $(M, g)$ are all strictly greater than $2 \pi$.

Moreover $P$ is uniquely determined. 


\section{Approximating by compact Polyhedra}

Since the polyhedron $P_{\theta}$ is not compact, we will find a family of compact hyperbolic polyhedra $P_{\theta}^{t}, t>0$ approximating $P_{\theta}$ by applying the above result. We define spherical complexes $S_{\theta}^{t}$ by modifying $S_{\theta}$ so that it is the dual of a compact hyperbolic polyhedron as follows. We increase the length of every edge of $S_{\theta}$ which does not belong to $B$ by a factor of $(1+t)$ and add a vertex $v_{i}$ at the pole of each hemisphere $H_{i}$, and join $v_{i}$ by an edge to each vertex on the equator of $H_{i}$. Note that if we let $t=0$ then $S_{\theta}^{0}=S_{\theta}$. It will be shown, using the above characterization (theorem 4), that $S_{\theta}^{t}$ is the dual of a compact hyperbolic polyhedron $P_{\theta}^{t}$. Furthermore $P_{\theta}^{t}$ has combinatorial type of $P_{\theta}$ with its ideal vertices truncated and the truncating faces are perpendicular to the faces of $P_{\theta}^{t}$ intersecting it. Thus in the Klein model, all the planes intersecting a truncating face meet in a single point outside $S_{\infty}^{2}$ corresponding to the unique point perpendicular to the truncating face. A generalized hyperbolic polyhedron is when we allow vertices to be outside $S_{\infty}^{2}$ in the Klein model and we call the vertices outside of $S_{\infty}^{2}$ hyper-ideal. In this paper we will not distinguish between a generalized polyhedron and the unique polyhedron obtained by truncating the hyper-ideal vertices. Thus $P_{\theta}^{t}$ corresponds to a generalized hyperbolic polyhedron with combinatorial type $P_{\theta}$ and with the vertices corresponding to ideal vertices of $P_{\theta}$ being hyper-ideal. Intuitively as $t$ tends to 0 these hyper-ideal vertices tend to $S_{\infty}^{2}$ and become ideal.

Lemma: 1 Given $\theta, 0<\theta \leq \pi$ then there exists $\delta>0$ such that the spherical complex $S_{\theta}^{t}$ is the dual of a unique compact hyperbolic polyhedron $P_{\theta}^{t}$ for $0<t<\delta$.

Proof :Obviously $S_{\theta}^{t}$ is a spherical complex satisfying (a) and (b) of the characterization (theorem 4 ), and therefore we need only show that there are no geodesics of length $\leq 2 \pi$. Let $\gamma^{t}$ be a geodesic in $S_{\theta}^{t}$. If $\gamma^{t}$ intersects the interior of a hemisphere $H$ in a single arc then $l\left(\left.\gamma^{t}\right|_{H^{o}}\right)=\pi$. Therefore if $\gamma^{t}$ intersects more than two hemispheres in their interiors then $l\left(\gamma^{t}\right) \geq 3 \pi$.

Case 1: $\gamma^{t}$ doesn't intersect the interior of bigon $B$. Then we get a curve in $S_{\pi}^{t}$ by removing the bigon and stitching back the edges. This curve we also call $\gamma^{t}$ and it is a geodesic in $S_{\pi}^{t}$.

If $\gamma^{t}$ intersects the interior of two hemispheres $H_{1}, H_{2}$ and if $\gamma^{t}$ doesn't contain an edge then $H_{1}, H_{2}$ intersect in points a distance $\geq \pi$ apart giving a contradiction. Thus $\gamma^{t}$ must contain an edge and $l\left(\gamma^{t}\right) \geq 2 \pi+\pi / 2>2 \pi$.

If $\gamma^{t}$ intersects the interior of only one hemisphere $H$, then it must enter and leave $H$ along an edge. We replace the arc in $H^{\circ}$ by an edge path of the same length by going along the boundary of $H$. This gives us a new geodesic which we still call $\gamma^{t}$ satisfying

$$
l\left(\gamma^{t}\right) \geq \pi / 2+\pi / 2+\pi / 2(1+t)+\pi / 2(1+t)=2 \pi+t \pi>2 \pi
$$

Therefore all geodesics in $S_{\theta}^{t}$ not intersecting the interior of $B$ have length bounded away from $2 \pi$.

Case 2: $\gamma^{t}$ intersects interior of $B$. There are a number of possibilities. 
If $\gamma^{t}$ enters $B$ at either the apex of $T_{1}$ or $T_{2}$ then it has to leave at the other apex and $l\left(\left.\gamma^{t}\right|_{B^{\circ}}\right)=\pi$. We replace this arc by edge path $e_{1}^{*} \cup e_{2}^{*}$ or $\bar{e}_{1}^{*} \cup \bar{e}_{2}^{*}$ depending on which gives a geodesic as a result (at least one must give a geodesic). Thus we have a geodesic which has the same length as $\gamma^{t}$ and doesn't intersect the interior of $B$. Therefore $\gamma^{t}$ has length $>2 \pi$ by the first case.

If $\gamma^{t}$ intersects the interior of two hemispheres $H_{1}, H_{2}$ and both are not incident to $B$ then $\gamma^{t}$ must contain an edge of $S_{\theta}^{t}$ and therefore $l\left(\gamma^{t}\right) \geq 2 \pi+\pi / 2>2 \pi$. If either $H_{1}, H_{2}$ are incident to $B$ only at an apex then $\gamma^{t}$ intersects $B$ in an arc from one apex to the other and has been already shown to be $>2 \pi$. Therefore either $\gamma^{t}$ contains an edge and is longer than $2 \pi$ or $H_{1}, H_{2}$ intersect at a point $p$ along their boundaries where $\gamma^{t}$ traverses from $H_{1}$ to $H_{2}$. But $H_{1}, H_{2}$ correspond to hemispheres in $S_{\pi}^{t}$ whose intersection is contained in $e_{1}^{*} \cup e_{2}^{*}$ and thus $H_{1}, H_{2}$ cannot intersect at point $p$. Therefore any geodesic in $S_{\theta}^{t}$ intersecting the interior of two or more hemispheres has length bounded away from $2 \pi$.

If $\gamma^{t}$ only intersects the interior of one hemisphere $H$ and it enters $B^{o}$ along an edge and leaves along an edge then it must intersect $B^{o}$ along the equatorial edge. Therefore there is a geodesic in $S_{\pi}^{t}$ of length $l\left(\gamma^{t}\right)-(\pi-\theta)$ obtained by removing the bigon as before and taking what is left of $\gamma^{t}$ as the curve. This must be of length $>2 \pi$, therefore $\gamma^{t}$ has length $\geq 2 \pi+(\pi-\theta)>2 \pi$. If $\gamma^{t}$ enters through $H$ and leaves along an edge at vertex $v$ of $B(v$ is not an apex), then we first replace the interior arc in $H$ by a boundary edge path $b$ of $H$ of the same length, we call this new curve $\gamma^{t}$ also. Now $\gamma^{t}$ contains two sides of a spherical triangle $\bar{T}$ in $B$. $H$ intersects $B$ in an edge $e$ and let $v_{1}$ be the vertex of $B$ contained in $b \cap e$. Now replace the part of $\gamma^{t}$ consisting of two sides of the spherical triangle $\bar{T}$ by the edge of $B$ joining $v, v_{1}$ (figure 4). This gives a geodesic of smaller length that is an edge path and therefore $l\left(\gamma^{t}\right)>2 \pi$.

If $\gamma^{t}$ intersects no hemisphere in its interior then $\gamma^{t}$ is an edge geodesic containing the equatorial edge of $B$. Removing the bigon we get a geodesic in $S_{\pi}^{t}$ of smaller length which implies $l\left(\gamma^{t}\right)>2 \pi$.

Since no geodesic of $S_{\theta}^{t}$ has length $\leq 2 \pi$, then $S_{\theta}^{t}$ is the spherical dual of a unique compact hyperbolic polyhedron $P_{\theta}^{t}$ (by theorem 4).

To find what is the combinatorial structure of $P_{\theta}^{t}$ we need to find a cell division of $S_{\theta}^{t}$ into convex spherical polygons by geodesics of length less than $\pi$ and whose endpoints are cone points. This is how we described $S_{\theta}^{t}$ originally, but this may not be the only cell division possible. To show that it is, consider the apex $v$ of one of the triangles $T_{1}, T_{2}$. The only other vertices a distance less than $\pi$ from $v$ are those vertices connected to $v$ by an edge in the original cell division and therefore the edges from $v$ must be a subset of the original edges from $v$. Also all edges must be included as if any are left out then there is an angle $\geq \pi$ subtended at $v$. Now considering a vertex $v_{1}$ on the equator we have an angle of $\pi$ inside the bigon $B$ at $v_{1}$. Therefore the equatorial edge of $B$ must also be included in the division. Using the fact that any geodesic intersecting the interior of a hemisphere must have length $\geq \pi$ and that no vertex can have an angle of $\geq \pi$ subtended at it, it is easy to show that all other edges of the original division are necessary. There cannot be any more edges as any 


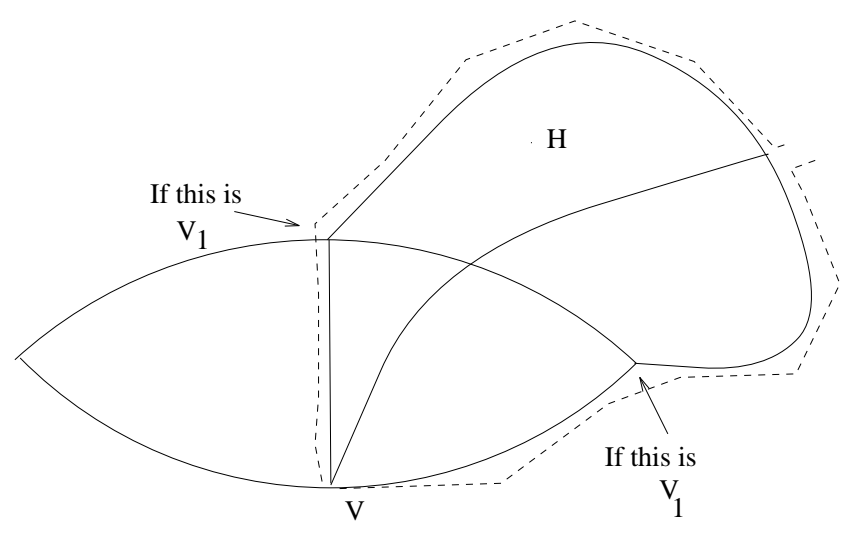

Figure 4: Replacing geodesic by edge geodesic

other edge from vertex to vertex would cross another edge in an interior point which can't happen. Therefore $P_{\theta}^{t}$ has dual given by the original cell division of $S_{\theta}^{t}$.

$P_{\theta}^{t}$ is a general hyperbolic polyhedron with combinatorial type $P \cup g$ and hyper-ideal vertices corresponding to the ideal vertices of $P$. The dihedral angles between a truncating plane and the planes it intersects are all $\pi / 2$. Also the dihedral angle along the edge corresponding to $g$ is $\theta$ and except for the four edges incident to $g$ which have dihedral angles $\pi / 2$, all others have dihedral angle $\pi / 2(1-t)$. Similarly the face angles on all truncating faces are $\pi / 2(1-t)$ except where the four edges incident to $g$ intersect. The face angles on the two faces perpendicular to $g$, at the vertices incident to $g$, are $\theta$, and all other face angles are $\pi / 2$. As $t$ tends to zero all dihedral angles approach $\pi / 2$ except along $g$ which has fixed dihedral angle $\theta$.

\section{Monotonicity and Volume Increase}

Lemma 1 shows that $P_{\theta}^{t}$ exists for $0<t \leq k(\theta), 0<\theta \leq \pi$ where $k(\theta)>0$. We can choose $k(\theta)=c \cdot \theta$ where $c$ is a positive constant. Hodgson and Rivin showed ([HR]) that the map from the space of compact hyperbolic polyhedra to their spherical duals is a homeomorphism and hence $P_{\theta}^{t}$ is a continuous two-parameter family of polyhedra in the above domain. When all the dihedral angles are acute we have continuity by Alexandrof ([A]) and therefore $\lim _{t \rightarrow 0} P_{\pi}^{t}=P$. Thus we extend $P_{\theta}^{t}$ by letting $P_{\pi}^{0}=P$. Also if $0<\theta \leq \pi / 2$ then $\lim _{t \rightarrow 0} P_{\theta}^{t}=P_{\theta}^{0}$ where $P_{\theta}^{0}$ is the unique hyperbolic polyhedron with combinatorial type of $P \cup\{g\}$ and dihedral angles $\pi / 2$ except along $g$ which has dihedral angle $\theta$. We can now take the limit as $(t, \theta)$ tends to $(0,0)$ and we get that $\lim _{(t, \theta) \rightarrow(0,0)} P_{\theta}^{t}=P^{\prime}$. Therefore $P_{\theta}^{t}$ is well defined and continuous on the triangle $0 \leq t \leq c \cdot \theta, 0 \leq \theta \leq \pi$ except for along the open interval of length $\pi / 2$ from $(0, \pi / 2)$ to $(0, \pi)$.

We now choose a continuous one-parameter family of polyhedra interpolating between $P$ and $P^{\prime}$ by taking the image of a piecewise linear path joining $(0, \pi)$ and $(0,0)$ in the domain 
of $P_{(\cdot)}^{(\cdot)}$ (figure 5). Thus the one-parameter family $X$ interpolating between $P$ and $P^{\prime}$ is the product of four continuous one-parameter families $X_{i}, i=1, \ldots, 4$ obtained by taking the image of the path joining the four points $(0, \pi),(0, t),(c / t, t),(c / t, 0),(0,0)$ by alternately horizontal and vertical lines.

The volume change from $P$ to $P^{\prime} \Delta V$ satisfies

$$
\Delta V=\Delta V_{1}+\Delta V_{2}+\Delta V_{3}+\Delta V_{4}
$$

where $\Delta V_{i}$ is the volume change over $X_{i}$.

By continuity given $\epsilon>0$ we can choose a $t>0$ such that

$$
\begin{aligned}
\left|\Delta V_{i}\right| & \leq \epsilon \quad i \neq 2 \\
\left|l^{t}(\pi)-l^{0}(\pi)\right| & \leq \epsilon
\end{aligned}
$$

where $l^{0}(\pi)=l$, the length of the original geodesic in $P$.

If $l^{t}$ is monotonic then

$$
\begin{aligned}
0 \leq \Delta V_{2} & \leq 1 / 2(\pi-t / c) l^{t}(\pi) \leq \pi / 2 \cdot l^{t}(\pi) \\
\Rightarrow 0 \leq \Delta V & \leq \pi / 2 \cdot l^{t}(\pi)+3 \epsilon \leq \pi / 2 \cdot l+\tilde{\epsilon}
\end{aligned}
$$

Therefore by choosing smaller values of $t$ we have

$$
\Delta V \leq \pi / 2 \cdot l
$$

Taking the four-fold cover of $P$ given by the hyperbolic link complement $L_{P}$ we have

$$
\Delta V^{\prime}=4 \cdot \Delta V \leq 4 \cdot(\pi / 2 \cdot l)=2 \pi l=\pi L
$$

where $\Delta V^{\prime}$ is the volume increase from $L_{P}$ to $L_{P^{\prime}}$ and $L$ is the length of the geodesic drilled from $L_{P}$ to obtain $L_{P^{\prime}}$.

\section{Monotonicity}

The following theorem is based on Cauchy Rigidity and is the step needed to prove monotonicity, but first a definition.

Definition: 1 A signed polyhedron $P$ is a polyhedron whose edges are labeled with either + , - or 0 .

A vertex $v$ of a signed polyhedron has type given by listing the signs on the edges incident to $v$ in a clockwise or anti-clockwise manner (with the usual equivalence) and a face $F$ is called a zero-face if all its edges are marked with zero. 


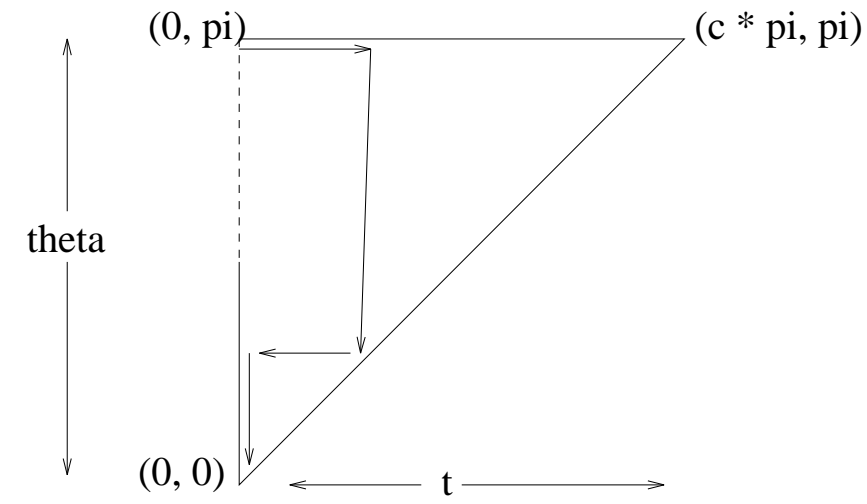

Figure 5: 2-parameter domain and path from $P$ to $P^{\prime}$

Theorem 5 Let $P$ be a trivalent signed polyhedron such that, except for two disjoint faces $F_{1}, F_{2}$, every face is either a zero-face or has at least four sign changes. Then the space obtained by collapsing all zero-edges $\tilde{P}$ is topologically a bouquet of spheres where either

(1) Each sphere has the trivial cell division (a point and a disc).

or

(2) There is exactly one sphere $\tilde{S}$ in the bouquet with a non-trivial cell division. $\tilde{S}$ has two cells with no sign changes and every other cell has exactly four sign changes. Also all vertices of $\tilde{S}$ have type $(++-),(--+)$ or $(+-+-)$ and therefore valence three or four.

Proof :Let $\Sigma$ be the set of edges of $P$ and $\Sigma=\Sigma_{+} \cup \Sigma_{-} \cup \Sigma_{0}$ be the partition into + , - and 0 edges. If $\left|\Sigma_{0}\right|=0$ then construct a vector field on $P$ as follows. In a neighborhood of $\Sigma$ the vector field is transverse to $\Sigma_{+}$and parallel to $\Sigma_{-}$. The index of a vertex $v$ can easily be calculated from its type. One way is to read the type of a vertex from left to right, count $-1 / 4$ for sign changes, $-1 / 2$ for same sign and then adding 1 . This is never positive if the valence is at least three and if the valence of $v$ is greater than four its index is always negative. In our case the vertices all have valence three but in general only vertices of type $(++-),(--+)$ and $(+-+-)$ (which have index zero), have non-negative indices (figure $6)$.

The vector field is extended over a face $F$ as follows. As we go round the edges of $F$ the sign changes $2 n$ times, which means that there are $n$ disjoint + segments joined together by $n$ disjoint - segments. Choose a center point $c_{F}$ in the interior of $F$ and for each + segment we join an interior point of it to the center $c_{F}$ by a prong and extend the vector field in a neighborhood of these $n$ prongs in the obvious way. Cutting along the prongs divides $F$ into $n$ pieces over which the vector field can easily be extended. The index of the critical point $c_{F}$ is $1-n / 2$ (figure 7 ). Note that if the face has no sign changes then the vector field can still be extended with $c_{F}$ having index 1 , in keeping with the formula. The interior of each face has at most one critical point and all other critical points are vertices of $P$. Each vertex contributes a non-positive index and each face $F \neq F_{1}, F_{2}$ has at least four sign changes, 


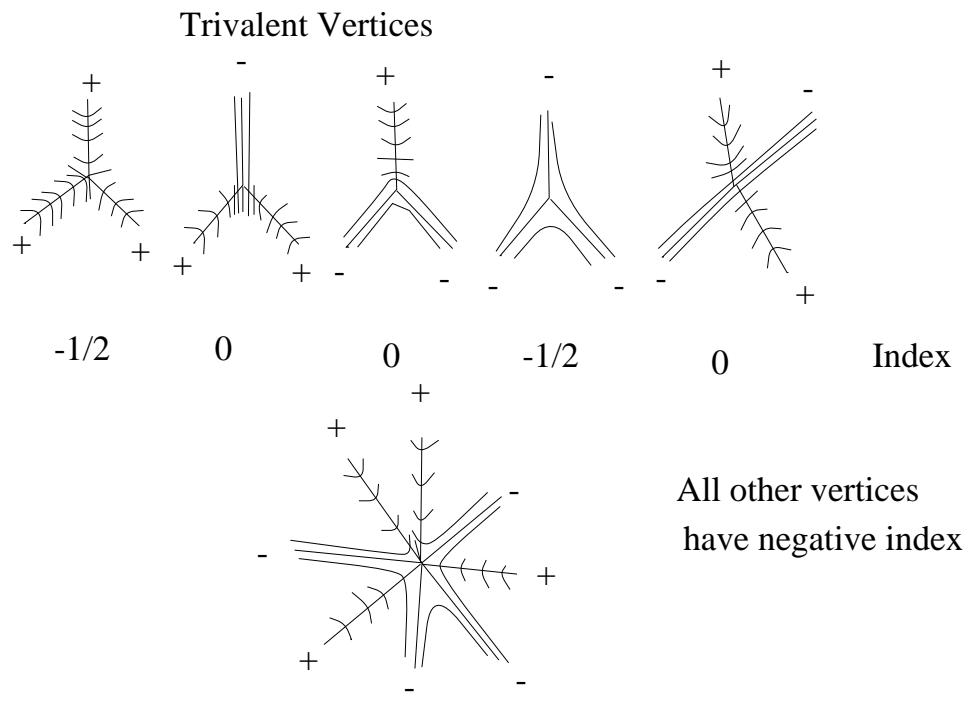

Typical vertex(index $-3 / 2)$

Figure 6: Indices of vertices

which implies $c_{F}$ has non-positive index. In order to have the sum of the indices equal to two (the Euler number of $P$ ), the indexes of both $c_{F_{1}}, c_{F_{2}}$ must be one and therefore they are of constant sign. Also all vertices and centers $c_{F}, F \neq F_{1}, F_{2}$ must have index zero. Therefore all vertices have type $(++-),(--+)$ and each $F$ has exactly four sign changes.

If $\left|\Sigma_{0}\right| \neq 0$ then we cannot find a vector field extending over $\Sigma_{0}$. We avoid this problem by collapsing these zero-edges. First consider $\bar{\Sigma}_{0} \subseteq \Sigma_{0}$ to be the collection of edges belonging to loops in $\Sigma_{0}$. This decomposes $P$ into regions $\left\{R_{i}\right\}$ which have disjoint interiors and are $n_{i}$-connected regions whose boundaries consist of zero-edges. Some regions correspond to a zero-face which we call zero-regions. To find a cell division with no zero-edges we must collapse $\Sigma_{0}$. Each region $R_{i}$ becomes a sphere $\tilde{S}_{i}$ in a bouquet of spheres $\tilde{P}$. The zero-regions become spheres with the trivial cell division of a point and a disc and every other face $F$ collapses to a cell $\tilde{F}$ of some $\tilde{S}_{i}$.

We collapse $\Sigma_{0}$ by first collapsing $\bar{\Sigma}_{0}$. Each $R_{i}$ becomes a sphere $S_{i}$. If $R_{i}$ is not a zeroface then we have each component of $\partial R_{i}, \beta_{i j}$ collapses to a vertex $v_{i j}$ on a sphere $S_{i}$. As $R_{i}$ is not a face there is an edge path $\gamma_{i j}$ splitting $R_{i}$ into two regions $R_{i j}^{1}, R_{i j}^{2}$ with endpoints on $\beta_{i j}$. If there are no other paths from $\beta_{i j}$ to the interior then either $R_{i}$ is the union of two faces or there are two faces which intersect in two separate edges (figure 8). Both give contradictions. Therefore collapsing $\beta_{i j}$ gives vertex $v_{i j}$ with valence at least three. Thus the vertices on $S_{i}$ after collapsing $\bar{\Sigma}_{0}$, have valence at least three. If we now collapse $\Sigma_{0}-\bar{\Sigma}_{0}$ to get sphere $\tilde{S}_{i}$ in $\tilde{P}$ the valences cannot go down as the only zero-edges left on $S_{i}$ form a collection of disjoint trees.

Therefore if $\tilde{S}_{i}$ does not correspond to a zero-face it has a decomposition into faces all but two of which have at least four sign changes and all vertices have valence at least three. Thus 


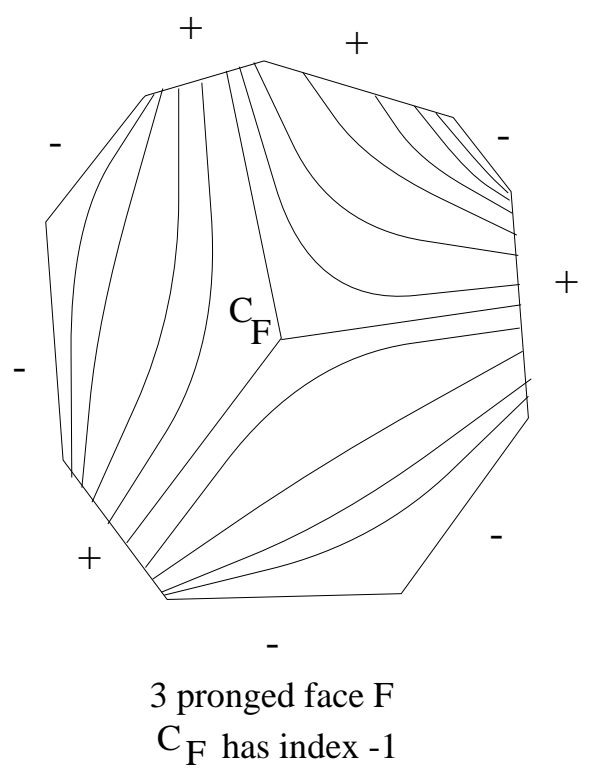

Figure 7: Extend vector field over face

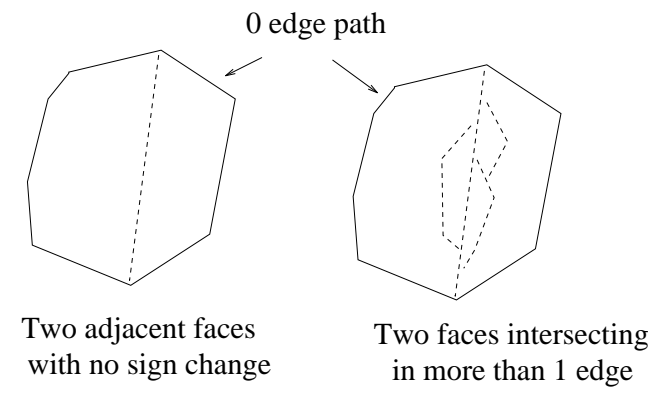

Figure 8: Valence after collapse is at least three 
as before we can give $\tilde{S}_{i}$ a vector field which implies that there can be at most one sphere $\tilde{S}$ which has a non-trivial cell division. On $\tilde{S}$ the vertices all have type $(++-),(--+)$ or $(+-+-)$, the faces corresponding to $F_{1}, F_{2}$ give rise to faces $\tilde{F}_{1}, \tilde{F}_{2}$ of $\tilde{S}$ which have constant sign on their boundaries and all other faces have exactly four sign changes.

Now we apply the above theorem. If we compare the corresponding faces of $P_{\theta_{1}}^{t}$ and $P_{\theta_{2}}^{t}$ then apart from the two faces $F_{1}, F_{2}$ which are incident and perpendicular to $g$, all others have identical face angles. Let $Q$ be the marked polyhedron combinatorially equivalent to both $P_{\theta_{1}}^{t}, P_{\theta_{2}}^{t}$ and marked with + , - or 0 depending on whether the length of the edge in $P_{\theta_{1}}^{t}$ is bigger, smaller or equal in length to the corresponding edge of $P_{\theta_{2}}^{t}$. By the below result in planar hyperbolic geometry (lemma 2), any face $F \neq F_{1}, F_{2}$ is either a zero-face or has at least four sign changes. Therefore by the above (theorem 5 ), both faces $F_{1}, F_{2}$ have no sign changes. If $\theta_{2}>\theta_{1}$ then again using the below lemma, both faces must have constant sign + . If the edge $g$ is + then we get two $(+++)$ vertices and if the edge $g$ is 0 then after collapsing we get a $(++++)$ vertex, both giving contradictions.

Therefore $g$ must be marked with - and thus $l^{t}$ is monotonic in $\theta$.

Theorem 5 gives more information about the pattern on a marked polyhedron $P$. The region $R$ corresponding to $\tilde{S}$ is obtained by removing the zero-faces of $P$ and has no zeroloops with interior edges. When we collapse the boundary curves of $R$ to get sphere $S$ then any vertex of $S$ has valence three or four. Any zero-edge path of more than one edge will give a vertex in $\tilde{S}$ with valence greater than four and therefore every zero-edge in $S$ is isolated. These isolated zero-edges in $S$ must join two trivalent vertices which have alternating signs as you go around a neighborhood of the zero-edge.

In the case of the markings on $Q$ we obtain figure 9 .

The following is the technical lemma needed above. A proof can be found in the referenced paper.

Lemma: 2 ([HR]) Let $E_{1}, E_{2}, \ldots, E_{n}$ be lines in $H^{2}$ defining a convex polygonal curve $C$. Let $A_{i}=E_{i} \cap E_{i+1}(i=1, \ldots, n-1)$ be the vertices of $C$, let $\nu_{i}=E_{i}^{\perp}(i=1, \ldots, n)$ be the outward unit normals to the Minkowski inner product $\langle\cdot, \cdot\rangle$, and let $l_{i}$ be the length of $E_{i} \cap C=A_{i-1} A_{i}(i=1, \ldots, n-2)$. Let $E_{1}^{\prime}, E_{2}^{\prime}, \ldots, E_{n}^{\prime}$ be lines in $H^{2}$ defining another such curve $C^{\prime}$ with $A_{i}^{\prime}, \nu_{i}^{\prime}, l_{i}^{\prime}$ defined as above. Assume that

(i) $<A_{i}=<A_{i}^{\prime}$ for $i=2, \ldots, n-1$ and

(ii) $l_{i}^{\prime} \geq l_{i}$ for $i=2, \ldots, n-1$.

Then

$$
\left\langle\nu_{1}^{\prime}, \nu_{n}^{\prime}\right\rangle \leq\left\langle\nu_{1}, \nu_{n}\right\rangle
$$

with equality if and only if $A_{1} A_{2} \ldots A_{n-1}$ is congruent to $A_{1}^{\prime} A_{2}^{\prime} \ldots A_{n-1}^{\prime}$.

An easy consequence of this lemma is the following. Let $F_{a}, F_{b}$ be two hyperbolic $n$-gons with face angles equal. If we label an $n$-gon $F$ with,+ 0 or - by comparing lengths of corresponding sides of $F_{a}, F_{b}$, then either $F$ has all sides marked with 0 , in which case $F_{a}, F_{b}$ 


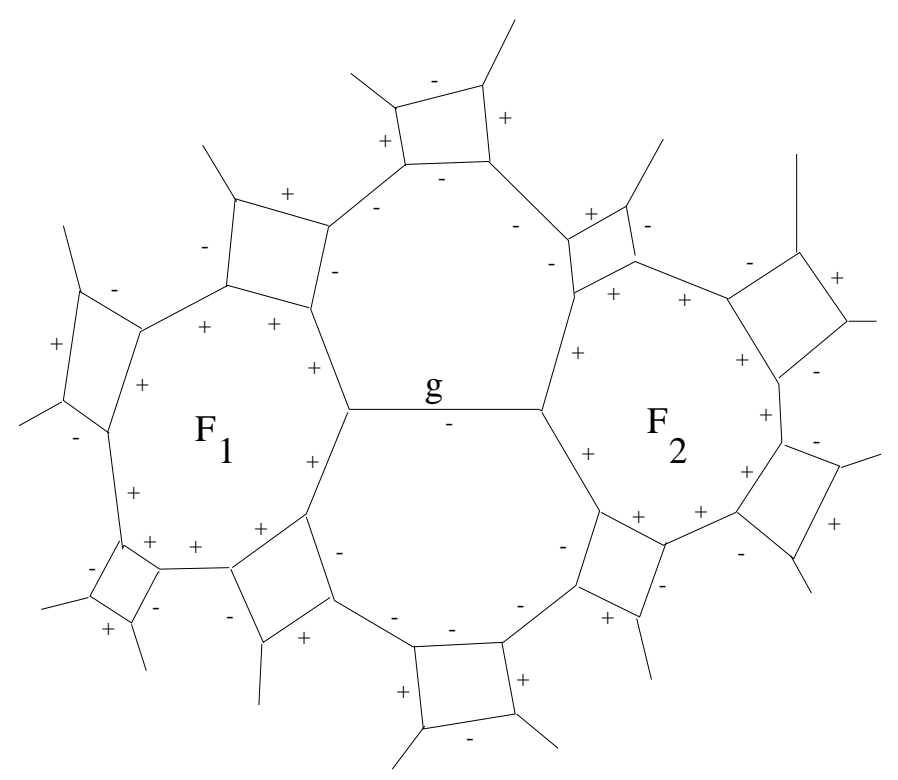

Figure 9: How edge lengths in $P_{\theta}^{t}$ vary with $\theta$

are congruent, or, $F$ has at least four sign changes as we go around the perimeter. This shows that $Q$ satisfies the hypothesis of the theorem.

To show that $F_{1}, F_{2}$ are marked + we need to relate the Minkowski inner product to face angles. If two edges of a compact hyperbolic polygon intersect in an angle $\theta<\pi$ then the inner product of their outward normals is $\cos \left(\theta_{\text {ext }}\right)$ where $\theta_{\text {ext }}$ is the exterior angle of $\theta$. Now the convex polygons $p_{1}, p_{2}$ corresponding to $F_{1}$ in $P_{\theta_{1}}^{t}, P_{\theta_{2}}^{t}$ respectively, have the same face angles except at the vertex incident to $g$, where $p_{i}$ has the angle $\theta_{i}$. Thus if the sides of $p_{1}$ where all smaller or equal in length to the sides of $p_{2}$ then we would have by the above lemma that the inner product of the outward normals of the two sides intersecting at $g$ in $p_{1}$ is greater than or equal to the same inner product in $p_{2}$, that is $\cos \left(\pi-\theta_{1}\right) \geq \cos \left(\pi-\theta_{2}\right)$. But $\theta_{1}<\theta_{2}$, and therefore the sides of $p_{1}$ must be greater than or equal in length to the corresponding sides in $p_{2}$. This gives the needed step to show that both $F_{1}, F_{2}$ are of constant sign + .

\section{Combinatorial Bound on Volume Increase}

Here we will show that bending along a geodesic $g$ on a face $F$ which is perpendicular to two edges of $F$ increases volume at most linearly in the number of edges of $F$.

The idea is that as $\theta$, the exterior dihedral angle at $g$ varies, $F$ is still intrinsically an ideal n-gon. If $l(\theta)$ is long then this forces $F$ to be narrow somewhere across $g$. This implies that the two faces $F_{1}, F_{2}$ obtained by bending $F$ along $g$ (which meet with exterior dihedral angle $\theta$ ), have faces $F_{3}, F_{4}$ adjacent to $F_{1}, F_{2}$ respectively which are connected by a short 
path on the bent face $F$. As $F_{3}, F_{4}$ cannot intersect, this shows that $l(\theta)$ must decrease as we bend otherwise $F_{3}, F_{4}$ would be forced to intersect.

We will describe the proof for when we have a continuous one-parameter family of polyhedra $P_{\theta}$ interpolating between $P$ and $P^{\prime}$. We obtain the result in general by approximating as before by the one-parameter family $X^{t}$.

\section{Long Geodesics give Thin Polygons}

An ideal hyperbolic quadrilateral has two perpendiculars and it can be shown using elementary hyperbolic geometry that the hyperbolic sine's of the half lengths of these are reciprocal. The following lemma is a generalization of this fact.

Lemma: 3 If $F$ is an ideal hyperbolic n-gon and $g$ the unique perpendicular to edges $e_{1}, e_{2}$ of $F$ then $\exists e_{3}, e_{4}$ separated by $g$ with distance $d$ between them satisfying

$$
\sinh (d / 2) \cdot \sinh \left(\frac{(\pi-2) l}{2 \pi(n-3)}\right) \leq 1
$$

where $l$ is the length of $g$.

Proof :Choose $\epsilon>0$ such that the one-sided $\epsilon$-neighborhood of $g$ has the same area as $F$.

$$
\Rightarrow l \sinh (\epsilon)=(n-2) \pi
$$

If we take $N_{\epsilon}$ the two-sided $\epsilon$-neighborhood of $g$ then for each $x \in g$ there is an arc $\gamma_{x}$ perpendicular to $g$ centered at $x$ of radius $\epsilon$. We define three subsets of $g$ as follows:

$$
g_{i}=\left\{x \in g:\left|\gamma_{x} \cap \partial F\right|=i\right\}
$$

Therefore $l=l_{0}+l_{1}+l_{2}$ where $l_{i}=$ length of $g_{i}, i=0,1,2$.

Each of the sets $g_{i}$ is a collection of intervals. If $A_{1}=$ Area of $F$ outside $N_{\epsilon}$ (cusp region) and $A_{2}=$ area of $F$ consisting of points whose perpendicular geodesic to $g$ has length less than $\epsilon$ (narrow region) then

$$
\begin{aligned}
\operatorname{Area}(F) & =\operatorname{Area}\left(F \cap N_{\epsilon}\right)+A_{1} \\
\operatorname{Area}\left(F \cap N_{\epsilon}\right) & =2 l_{0} \sinh (\epsilon)+l_{1} \sinh (\epsilon)+A_{2} \\
\Rightarrow l \sinh (\epsilon) & =2 l_{0} \sinh (\epsilon)+l_{1} \sinh (\epsilon)+A_{1}+A_{2} \\
\Rightarrow l_{2} \sinh (\epsilon) & =l_{0} \sinh (\epsilon)+A
\end{aligned}
$$

where $A=A_{1}+A_{2}$ (cusp plus narrow region).

If $e$ is one of the $n-2$ edges of $F$ not intersecting $g$, drop perpendiculars to $g$ from the endpoints of $e$ on $S_{\infty}^{1}$ to the two points $x, y$ on $g$. This forms a quadrilateral $Q_{e}$ with area $\pi$ and base $[x, y]$. Let $A_{e}$ be the area of the region in $Q_{e}$ which is either a distance greater than $\epsilon$ from $g$ or whose perpendicular to $g$ has length less than $\epsilon$. $A_{e}$ is the contribution of 


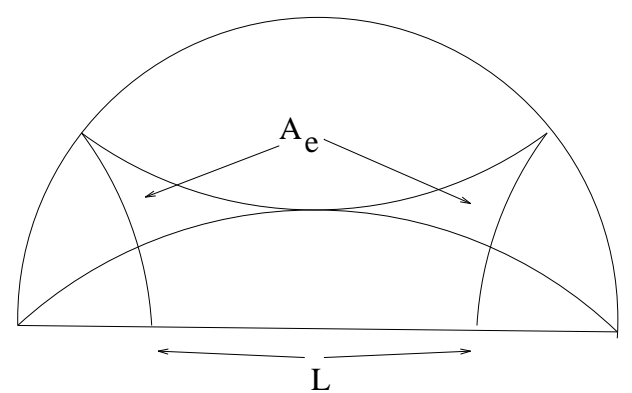

Figure 10: $A_{e}$ is minimum

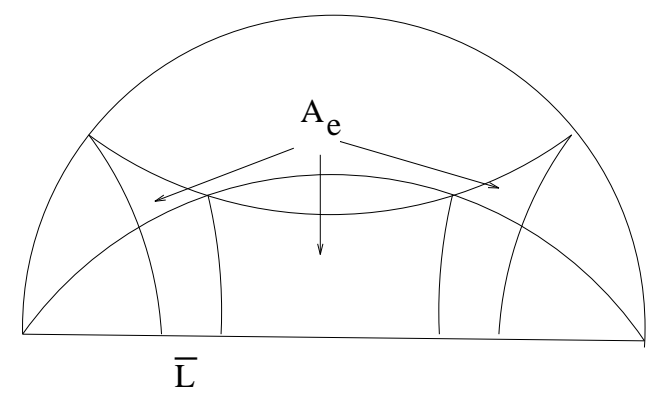

Figure 11: General picture of $A_{e}$

$Q_{e}$ to $A$. If $e$ doesn't intersect $N_{\epsilon}$ then the $A_{e}$ is minimum when $e$ is tangent to $\partial N_{\epsilon}$ (figure 10). If $L$ is the length of the base of the quadrilateral tangent to $\partial N_{\epsilon}$ then

$$
\begin{aligned}
\sinh (L / 2) & =\frac{1}{\sinh (\epsilon)} \\
\Rightarrow \quad A_{e} & \geq \pi-L \sinh (\epsilon)=\pi-\frac{L}{\sinh (L / 2)} \\
\Rightarrow \quad A_{e} & \geq \pi-2
\end{aligned}
$$

If $e$ intersects $\partial N_{\epsilon}$ at points $x^{\prime}, y^{\prime}$ then project down onto $g$ to get points $x_{1}, y_{1}$ (figure 11). Now if $\bar{L}$ is the length of either $\left[x, x_{1}\right]$ or $\left[y_{1}, y\right]$ (both have the same length) then

$$
\begin{aligned}
\bar{L} & \leq L / 2 \\
\Rightarrow A_{e} & =\pi-2 \bar{L} \sinh (\epsilon) \\
\Rightarrow A_{e} & \geq \pi-2 \\
\Rightarrow A & \geq(n-2)(\pi-2)
\end{aligned}
$$


Since we have that $l_{2} \sinh (\epsilon)=l_{0} \sinh (\epsilon)+A$ then

$$
\begin{aligned}
l_{2} & \geq \frac{A}{\sinh (\epsilon)} \geq \frac{(n-2)(\pi-2)}{\sinh (\epsilon)} \\
\Rightarrow l_{2} & \geq \frac{\pi-2}{\pi} \cdot l
\end{aligned}
$$

The subset $g_{2}$ consists of at most $(n-3)$ intervals. Therefore by the above, one of these intervals $[a, b]$ must have length $D$ satisfying

$$
D \geq \frac{\pi-2}{\pi(n-3)} \cdot l
$$

Consider the quadrilateral $Q$ formed by taking the two perpendicular arcs $\gamma_{a}, \gamma_{b}$ to $g$, centered at $a, b$ respectively, of length $2 \epsilon$. Choose the other sides $\alpha, \beta$ to join the endpoints of $\gamma_{a}, \gamma_{b}$. There are two edges $e_{3}, e_{4}$ of $F$ separated by $g$, which enter and leave $Q$ through the sides $\gamma_{a}, \gamma_{b}$. To show that $e_{3}, e_{4}$ are close note that they are closer than $\alpha$ and $\beta$ are. The two geodesics $\alpha, \beta$ describe an ideal quadrilateral by extending $\alpha, \beta$ to the sphere at infinity. This quadrilateral has a diagonal of length $D^{\prime}$ which is longer than $D$ whose other diagonal has length $d^{\prime}$. If $d$ is the distance between $e_{3}$ and $e_{4}$ then

$$
\sinh (D / 2) \leq \sinh \left(D^{\prime} / 2\right)=\frac{1}{\sinh \left(d^{\prime} / 2\right)} \leq \frac{1}{\sinh (d / 2)}
$$

Therefore we have

$$
\sinh (d / 2) \cdot \sinh \left(\frac{(\pi-2) l}{2 \pi(n-3)}\right) \leq 1
$$

This lemma shows that long geodesics must give thin polygons.

\section{Thin Bent Plane Forces Intersection}

In the hyperbolic plane consider a geodesic $\gamma$ represented by a circle $C$ of radius $R$ intersecting the unit circle at right angles. The two geodesics passing through the origin $o$ meeting $\gamma$ on $S_{\infty}^{1}$ form an angle of $\phi$ at $o$ (visual angle). If $x$ is the Euclidean distance from $o$ to $\gamma$ and $d$ is the hyperbolic distance then we have by simple planar geometry

$$
\begin{array}{rlrl} 
& & =\frac{1-x^{2}}{2 x} \\
& \tan (\phi / 2) & =R \\
& 2 \tanh ^{-1}(x) & =d \\
\Rightarrow \quad & x & & \tanh (d / 2) \\
\Rightarrow \quad & R & & \frac{1}{\sinh (d)} \\
\Rightarrow \quad \tan (\phi / 2) & =\frac{1}{\sinh (d)}
\end{array}
$$




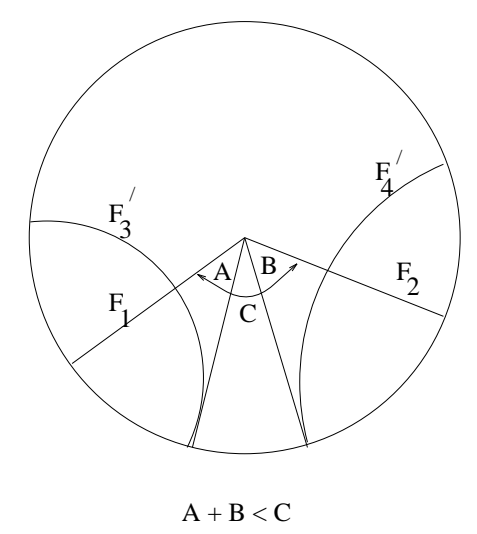

Figure 12: Sideview of the planes

In $P_{\theta}$ we have planes $F_{1}, F_{2}$ intersecting with dihedral angle $\phi$ and let $F_{3}, F_{4}$ be the two planes intersecting in edges $e_{3}, e_{4}$ respectively of lemma 3 . We also have planes $F_{3}^{\prime}, F_{4}^{\prime}$ defined by geodesics $\alpha, \beta$ which intersect $F_{1}, F_{2}$ at right angles.

If $F_{3}^{\prime}, F_{4}^{\prime}$ intersect then $F_{3}, F_{4}$ must also intersect. Therefore since planes $F_{3}, F_{4}$ cannot intersect then neither can $F_{3}^{\prime}, F_{4}^{\prime}$. Looking along the geodesic $g, F_{3}^{\prime}, F_{4}^{\prime}$ are given by two geodesics $\gamma_{3}, \gamma_{4}$ and $F_{3}^{\prime}, F_{4}^{\prime}$ by two geodesics passing through the origin and perpendicular to $\gamma_{3}, \gamma_{4}$ respectively (figure 12).

Both $\gamma_{3}, \gamma_{4}$ have the same visual angle $\phi^{\prime}$. If they are not to intersect then

$$
\begin{aligned}
\phi^{\prime} / 2 & \leq \phi / 2 \\
\Rightarrow \tan \left(\phi^{\prime} / 2\right) & \leq \tan (\phi / 2) \\
\Rightarrow \frac{1}{\sinh \left(d^{\prime} / 2\right)} & \leq \tan (\phi / 2)
\end{aligned}
$$

where $d^{\prime}$ is the length of the short curve on $F$ joining $F_{3}^{\prime}, F_{4}^{\prime}$. By lemma 3 we have

$$
\sinh \left(\frac{(\pi-2) l(\phi)}{2 \pi(n-3)}\right) \leq \tan (\phi / 2)
$$

Therefore

$$
l(\phi) \leq \frac{2 \pi(n-3)}{(\pi-2)} \cdot \sinh ^{-1}(\tan (\phi / 2))
$$

Therefore we obtain a combinatorial bound on the volume increase by integrating the above equation. This integration is possible as although $\sinh ^{-1}(\tan (\phi / 2))$ tends to infinity at $\pi$ it is still integrable. Therefore we have

$$
\Delta V \leq \frac{\pi(n-3)}{(\pi-2)} \cdot \int_{0}^{\pi} \sinh ^{-1}(\tan (\phi / 2) d \phi=K \cdot(n-3)
$$

where $K$ is some fixed constant. We can derive this bound in general using the one-parameter family of approximations $X^{t}$ in the same manner as before. 


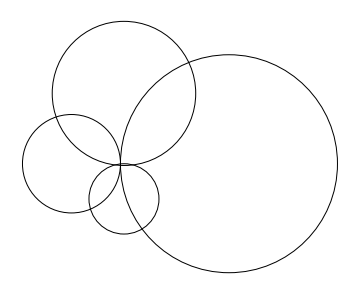

Figure 13:

\section{Non-existence of lower bound for volume increase}

If the volume increase under Dehn drilling is bounded below by a constant times some positive power of the length of the geodesic drilled then we have that

$$
\Delta V \geq c \cdot l^{a}
$$

where $c, a$ are fixed positive constants and $l$ is the length of the geodesic drilled. We have shown in the previous section, that the volume increase by drilling out a geodesic belonging to an ideal $n$-gon of a basic polyhedron is bounded linearly in $n$. Therefore to prove that no lower bound exists we need to find a sequence of basic polyhedra each containing a face of bounded valence and the set of lengths of geodesics perpendicular to two sides unbounded. Let $\left\{P_{k}\right\}_{k=0}^{\infty}$ be a sequence of basic polyhedra and $N$ a number such that each $P_{k}$ has a marked face $F_{k}$ which is an $n_{k}$-gon, $n_{k}<N$. Also let $l_{k}$ be the length of the longest geodesic $\alpha_{k}$ in $F_{k}$ perpendicular to two edges of $F_{k}$. Then the volume increase $\Delta V_{k}$, by drilling out $\alpha_{k}$ is bounded above by some constant $K$ depending only on $N$. If the sequence $l_{k}$ is not bounded then by the lower bound for volume increase $\Delta V_{k} \geq c \cdot l_{k}^{a}$. Therefore $\Delta V_{k}$ is unbounded giving a contradiction. We now construct the required family using circle packings.

\section{Circle Patterns and Packings}

If $P$ is a hyperbolic polyhedron then in the upper half-space model each face $F$ of $P$ lies on a hemisphere $H_{F}$ which intersects $S_{\infty}^{2}$ in a circle $C_{F}$. Furthermore the angle at which two circles $C_{F_{1}}, C_{F_{2}}$ intersect equals the dihedral angle between the faces $F_{1}, F_{2}$. Thus looking for a polyhedron of prescribed dihedral angles and combinatorics is equivalent to finding a circle pattern with prescribed angles of intersection and combinatorial pattern. We will use the terminology circle packing to refer to a collection of circles with intersections being tangential.

In the case of constructing a basic polyhedron we require that the circles meet in groups of four with opposite circles tangent and neighboring circles meeting at right-angles (figure 13).

We start our circle pattern with a circle $C_{0}$ of radius one centered at the origin $O$. We further take circles $C_{1}, C_{1}^{\prime}$ of radius $r$ such that both are perpendicular to $C_{0}$ and have centers on the positive, negative real axis respectively. Thus there are unique circles $C_{2}, C_{2}^{\prime}$ which 
are perpendicular to $C_{0}$, with centers on the positive, negative imaginary axis respectively and are tangent to both $C_{1}, C_{1}^{\prime}$. This configuration of five circles depends only on our choice of $r$ and therefore we have a one-parameter family $\mathcal{C}_{r}$ of circle patterns. By simple planar hyperbolic geometry the radius of both $C_{2}, C_{2}^{\prime}$ is $1 / r$. This collection of five circles describes an ideal quadrilateral on the hyperbolic plane $H_{0}$ associated with circle $C_{0}$. Furthermore $r$ is a coordinate system for the shape of the quadrilateral with it becoming narrow in the horizontal direction as $r$ tends to infinity and becoming narrow in the vertical direction $r$ tends to 0 .

To extend $\mathcal{C}_{r}$ to the circle pattern of a basic polyhedron we perform a moebius transformation $M$ on $\mathcal{C}_{r}$. $M$ consists of a rotation of $\pi / 2$ about the origin followed by an expansion by a real number $k_{r}$. The factor $k_{r}$ is chosen so that $M\left(C_{1}\right)$ is tangent to the original $C_{1}$. For $r$ large these four tangent points are the only intersections between $\mathcal{C}_{r}$ and $M\left(\mathcal{C}_{r}\right)$ and so we extend circle pattern $\mathcal{C}_{r}$ by adding $M\left(\mathcal{C}_{r}\right)$ to form circle pattern $\overline{\mathcal{C}_{r}}=\mathcal{C}_{r} \cup M\left(\mathcal{C}_{r}\right)$ (figure 14).

Now take the collection of hemispheres $\mathcal{H}_{r}$ associated with $\overline{\mathcal{C}_{r}}$ and let $N_{r}$ be the region between the two hemispheres $H_{0}, M\left(H_{0}\right)$ cut out by $\mathcal{H}_{r} . N_{r}$ is an infinite volume hyperbolic orbifold with four infinite volume funnels. Each of these funnels meet $S_{\infty}^{2}$ in a quadrilateral bounded by circles which are tangent at points of intersection. It can be easily shown that all four of these quadrilaterals are equivalent up to moebius transformation to a quadrilateral $Q_{r}$.

To form a basic polyhedron from $N_{r}$ we must truncate the funnels of $N_{r}$. We do this by first finding a packing of $Q_{r}$ by a finite number of circles such that the interstices are all triangular. Then having packed each of the four copies of $Q_{r}$, we are left with a finite number of triangular interstices which we truncate by adding the unique circle passing through the three vertices of each interstice. These circles are also perpendicular to the three sides of the interstices and therefore we have truncated the four infinite volume funnels of $N_{r}$ to get a basic polyhedron $P_{r}$.

Unfortunately it is not always possible to find a finite packing of an arbitrary circular quadrilateral but using work of Robert Brooks $([\mathrm{Br}])$ we will show that there is a sequence $\left\{r_{n}\right\}$ such that $Q_{r_{n}}$ is packable and $r_{n}$ monotonically tends to infinity.

We define the continued fraction $c(Q)$ of a quadrilateral $Q$ by using the greedy algorithm. Assume that the four sides have been labelled left, right, top, bottom. We first pack $Q$ from left to right by starting at the left side of $Q$ and packing in the largest circle tangent to the left, top and bottom of $Q$. Now $Q$ contains a smaller quadrilateral $Q_{1}$ which we again pack with the largest circle tangent to the left, top and bottom sides. After $n_{1}$ circles have been added the remaining quadrilateral $Q_{n_{1}}$ is too narrow to fit any more circles which are tangent to the left, top and bottom so we now pack $Q_{n_{1}}$ from top to bottom with circles tangent to the top, left and right sides. Again after $n_{2}$ circles have been packed into $Q_{n_{1}}$ we are left with a quadrilateral $Q_{n_{1}, n_{2}}$ too wide to pack with a circle tangent to the top, left and right. The packing algorithm is defined recursively by now applying the algorithm to the quadrilateral $Q_{n_{1}, n_{2}}$ (figure 15). To each quadrilateral we get a sequence of integers $\left\{n_{i}\right\}$ and we define the function $c$ on the space of quadrilaterals to be the real number with these 


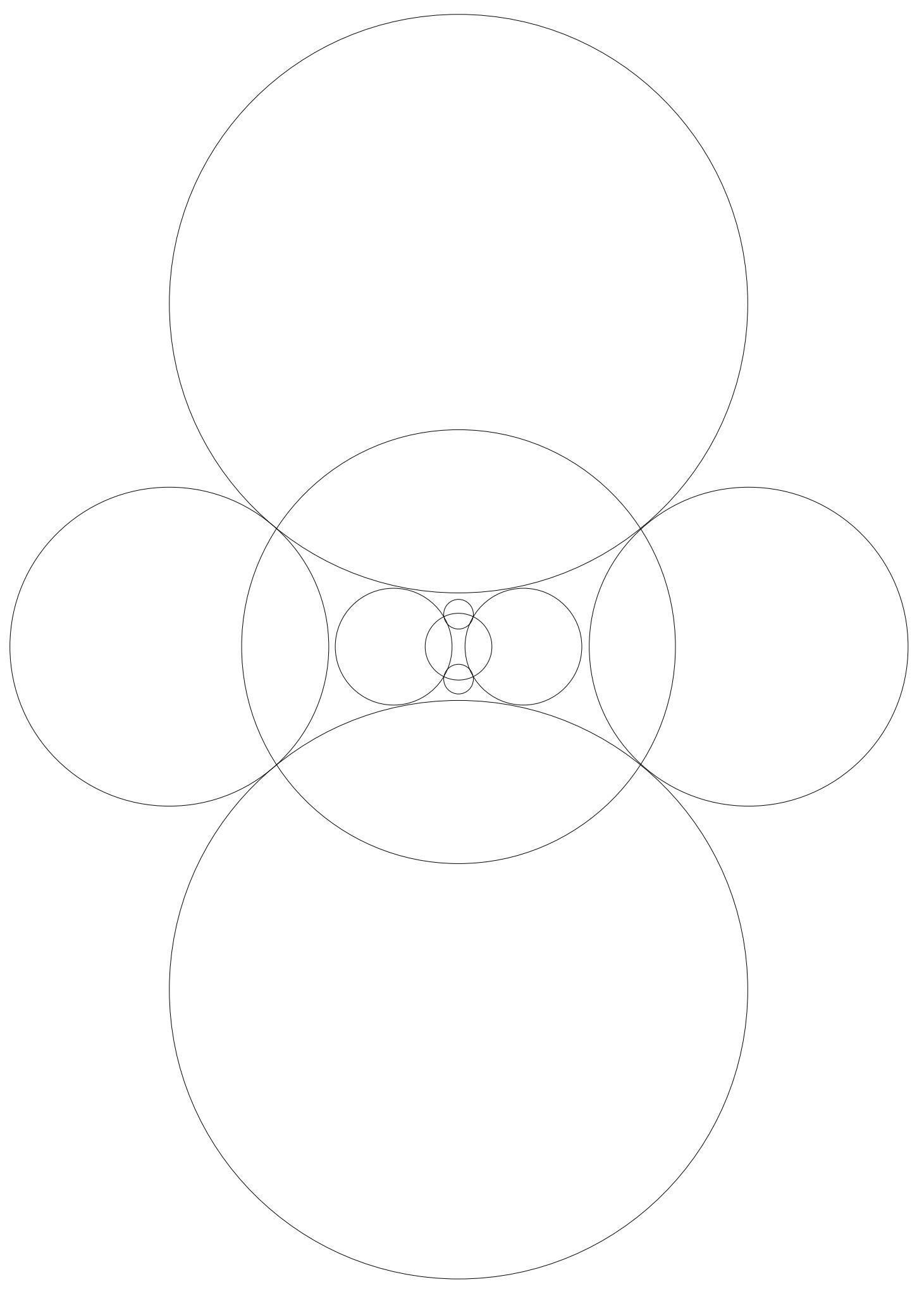

Figure 14: Circle pattern 


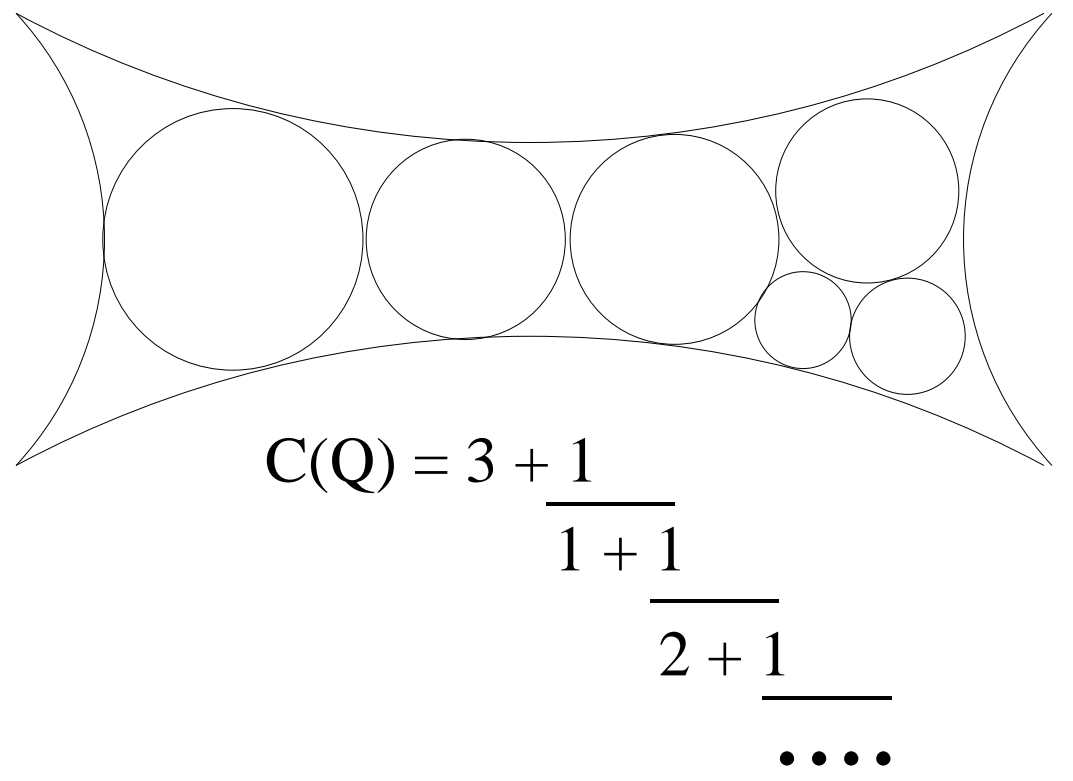

Figure 15: Greedy algorithm

as its continued fraction expansion, that is

$$
c(Q)=n_{1}+\frac{1}{n_{2}+\frac{1}{n_{3}+\frac{1}{n_{4}+\cdots}}}
$$

Note that the greedy algorithm gives a finite packing of $Q$ if and only if $c(Q)$ is rational. Now let $\mathcal{S}$ be the space of all configurations of four circles up to moebius transformation which have disjoint interiors and such that the complement of the interiors is two circular quadrilaterals. Then Robert Brooks showed $([\mathrm{Br}])$ that the map $\bar{c}: \mathcal{S} \rightarrow \Re^{2}$ defined by mapping a configuration of circles to the pair of continued fractions of its two circular quadrilateral regions, is not only continuous but a homeomorphism. This implies that $c$ is continuous and therefore $c\left(Q_{r}\right)$ is a continuous function of $r$. It can be shown that as $r$ tends to infinity the modulus of $Q_{r}$ tends to infinity. Therefore $c\left(Q_{r}\right)$ tends to infinity and by continuity there exists a sequence $\left\{r_{n}\right\}$ such that $c\left(Q_{r_{n}}\right)=n$. Therefore $Q_{r_{n}}$ can be packed with $n$ circles from left to right.

Now basic polyhedron $P_{n}$ is defined by truncating the funnels of $N_{r_{n}}$ as described above. Figure 16 is a combinatorial picture of $P_{n}$. The polyhedra $P_{n}$ have the property of each containing an ideal quadrilateral face $F_{n}$ whose modulus tends to infinity as a function in $n$. Thus $F_{n}$ contains a geodesic $\alpha_{n}$, perpendicular to two sides of $F_{n}$ whose length monotonically increases to infinity as a function of $n$. Therefore this sequence of polyhedra is a counterexample to the existence of a lower bound to volume increase under Dehn drilling. We get 
a similar counter-example for the manifold case by looking at the four-fold covers of these polyhedra when viewed as orbifolds.

\section{Conclusion}

The two bounds we derive are very different. One is a bound on the volume increase by the length of the geodesic drilled and the other is a purely combinatorial bound. I believe that these techniques can be applied to a wider class than I have done and further hope to prove the conjecture at the outset by decomposing the manifold into polyhedra and extending the results in this paper.

\section{References}

[A] Alexandroff Convex Polyhedra

[B1] M. Bridgeman Volume increase under Dehn drilling operations Phd. thesis Princeton June 1994

[B2] M. Bridgeman The Structure and Enumeration of Link Projections To Appear Trans. A.M.S. (1995)

[Br] R. Brooks On the Deformation Theory of Classical Schottky Groups Duke Mathematical Journal December 1985

[Th] W. P. Thurston The Geometry and Topology of 3-manifolds

[HR] Craig Hodgson, Igor Rivin A characterization of Compact Convex Polyhedra in Hyperbolic 3-Space

[Ho] Craig Hodgson Degeneration $\&$ Regeneration of Geometric structures on ThreeManifolds Phd. thesis Princeton June 1986 


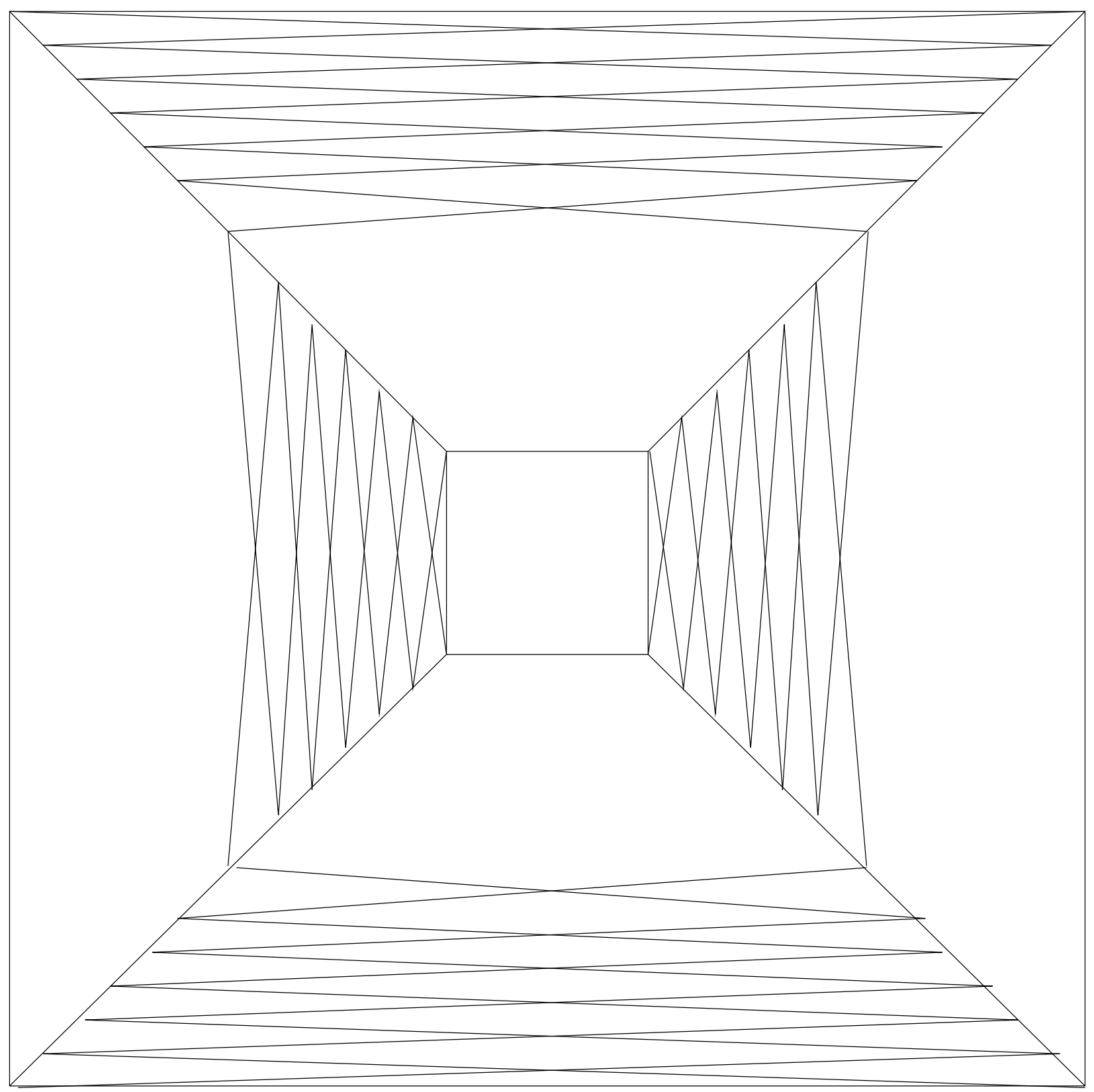

Figure 16: Polyhedron with thin ideal quadrilateral face 